\title{
Sistemas complexos: novas formas de ver a Botânica
}

\author{
GUSTAVO M. SOUZA ${ }^{1}$ e MARCOS S. BUCKERIDGE 2,3
}

(recebido: 13 de maio de 2004; aceito: 11 de agosto de 2004)

\begin{abstract}
Complex systems: new forms to see Botany). Part of the science field named Complexity has quickly been developing during the last 15 years and the application of such tools to the Plant Sciences is imminent. In the present review we present some of the basic concepts related to Complexity, and some examples of applications to different areas in Botany are given. The main reasoning of this work is that better understanding and subsequent application by the botanists of this approach to plant taxonomy, physiology, anatomy and ecology will probably lead to a considerable widening of what is known about plants.
\end{abstract}

Key words - atractor, Botany, complexity, fractals, neural networks

RESUMO - (Sistemas complexos: novas formas de ver a Botânica). Uma parte da ciência denominada Complexidade vem se desenvolvendo rapidamente durante os últimos 15 anos e as aplicações de tais ferramentas às Ciências Vegetais são iminentes. $\mathrm{Na}$ presente revisão, são apresentados conceitos básicos relacionados à Complexidade e dados alguns exemplos de suas aplicações em diferentes áreas da Botânica. O argumento principal deste trabalho é que a melhor compreensão e consequiente aplicação de tais enfoques à taxonomia, fisiologia, anatomia e ecologia pelos botânicos provavelmente levará a uma ampliação considerável do que se sabe sobre os vegetais.

Palavras-chave - atrator, Botânica, complexidade, fractais, redes neurais

\section{Introdução}

Assim como em outras áreas da ciência, a Botânica vem sofrendo uma lenta e, por vezes, imperceptível transformação. Não se trata de uma transformação relacionada às descobertas em sua própria área de abrangência, mas na forma como os cientistas aplicam métodos de outras áreas para fazer novas descobertas na Botânica. Para que tais transformações sejam apreciadas, é necessário compreender algumas das principais descobertas da matemática e suas consequiências durante os últimos 15 ou 20 anos. Na realidade, os pilares fundamentais para a constatação de que as relações entre as leis naturais poderiam ser bem diferentes do que se pensava haviam sido plantados bem antes. Cientistas como Georg Cantor (1845-1918), Gaston Julia (1873-1978) e Henri Poincaré (1854-1912) foram alguns dos

\footnotetext{
1. Universidade do Oeste Paulista, Campus II, Faculdade de Ciências Agrárias, Rod. Raposo Tavares km 572, 19067-175 Presidente Prudente, SP, Brasil.

2. Instituto de Botânica, Seção de Fisiologia e Bioquímica de Plantas, Caixa Postal 4005, 01061-970 São Paulo, SP, Brasil.

3. Autor para correspondência: msbuck@usp.br
}

responsáveis pela fundação desses alicerces. Foi por volta da década de 1960, graças ao advento de computadores mais eficientes e técnicas matemáticas mais refinadas, que Edward Lorenz percebeu que havia algo errado quando tentava fazer com que seu computador fizesse uma previsão do tempo. Ele percebeu que, ao dar entrada aos números iniciais para que as equações calculassem as probabilidades de ocorrência de eventos climáticos, números bem à direita da vírgula faziam uma diferença enorme. Lorenz compreendeu que pequenas diferenças eram fundamentais e daí surgiu o famoso signo do "efeito borboleta", ou seja, algo como: o bater das asas de uma borboleta no Brasil pode mudar o tempo na China. Em 1982, Benoit Mandelbrot, com a publicação do hoje clássico livro The fractal geometry of nature, pôs abaixo a idéia de que só existem dimensões geométricas inteiras (ponto com dimensão 0 , reta com dimensão 1, plano com dimensão 2, e assim por diante). Ele demonstrou que, em todos os lugares da natureza, existem dimensões fracionadas, ou seja, dimensões que variam entre duas dimensões inteiras $(1,2$ ou 2,3 por exemplo). Paralela e independentemente, Robert May, com a publicação de um artigo sobre complexidade em 1976 e posteriormente com outros importantes trabalhos, tem gerado descobertas desconcertantes 
sobre como o universo "não linear" funciona.

Tais descobertas acabaram se fundindo com as descobertas de Lorenz e Mandelbrot, gerando a idéia geral de que fenômenos naturais podem passar da linearidade para o caos e vice-versa, mas que o "caos" não seria tão desorganizado assim. Por isso tem sido chamado de caos determinístico ${ }^{5}$, para diferenciá-lo de fenômenos verdadeiramente aleatórios. Por outro lado, cada vez que um sistema passa a variar não linearmente (à maneira do caos determinístico), este deixa como rastro uma estrutura com dimensão fracionada (um fractal).

Uma característica importante dos sistemas não lineares é que equações extremamente simples podem gerar padrões extremamente complexos. Daí a relação com as descobertas de Lorenz.

Gradativamente, essas descobertas foram absorvidas e, com isso, a existência de dimensões fractais e fenômenos não lineares começaram a ser detectadas e apontadas em várias áreas das ciências, e a Botânica não é uma exceção. Uma estrutura anatômica, ao ser estudada, ainda possui as mesmas formas previamente descritas, desenhadas ou fotografadas pelos botânicos, mas o que muda quando se usam estas novas ferramentas é a interpretação de, por exemplo, como tal estrutura se desenvolveu ou de como ela foi moldada durante a evolução, ou ainda como tal estrutura afeta as relações daquele indivíduo com o seu ambiente. Na imensa maioria dos casos, nossas descrições de estruturas e mecanismos ainda são confortavelmente explicadas do ponto de vista estritamente linear e ainda que tais arcabouços intelectuais possam ser transpostos, no futuro, para visões mais amplas e modernas, as ferramentas intelectuais necessárias para implementar tal visão de fenômenos na área de ciências vegetais já estão disponíveis.

Acreditamos que tal visão permita uma apreciação mais aprofundada e mais próxima da realidade dos vegetais, de forma a possibilitar a compreensão e mesmo o estabelecimento de correlações imperceptíveis, quando usamos estritamente ferramentas do universo linear. Este

4. Sistemas não-lineares são aqueles em que a influência de um estado anterior do sistema dinâmico sobre o posterior não é diretamente proporcional, mas envolvem laços de retroalimentação que podem influenciar o sistema globalmente (interações não-locais).

5. Dinâmicas caóticas caracterizam os sistemas cujas trajetórias inicialmente próximas divergem exponencialmente com o passar do tempo em função de uma alta sensibilidade às condições iniciais, estes são ditos sistemas dinâmicos caóticos. artigo tem o objetivo de introduzir alguns dos conceitos relacionados à complexidade e mostrar, através de exemplos, como tal visão diferenciada pode ser obtida na área da Botânica.

\section{Caos, fractais e sistemas complexos: um novo paradigma}

Neste novo contexto teórico, o estudo dos sistemas ditos complexos tornou-se um aspecto central na ciência contemporânea. O desenvolvimento de teorias matemáticas dos sistemas dinâmicos não-lineares (May 1976, Hütt \& Lüttge 2002), teoria do caos (Ruelle 1989, Fiedler-Ferrara \& Prado 1994), e fractais (Mandelbrot 1982, Moreira 1999) trouxe novas possibilidades de observação e interpretação de dados biológicos, permitindo-nos uma maior aproximação da realidade complexa e dinâmica dos sistemas vivos.

Genericamente, sistemas complexos são aqueles compostos de muitos elementos e/ou subsistemas diferentes interagindo espacialmente e temporalmente de forma não linear, gerando padrões emergentes que são observáveis apenas em escalas maiores. Neste sentido, o termo complexidade refere-se à descrição dos estados de um sistema complexo. Existem diferentes medidas de complexidade. Algumas delas podem estar relacionadas simplesmente com a quantidade de diferentes elementos que compõem um sistema, o que é, na verdade, uma medida parcial, pois desconsidera as relações entre os elementos do sistema. Outras medidas estão relacionadas com o número e intensidade (grau de conectância) das relações entre os elementos do sistema, formando uma intrincada rede de relações. Outras medidas ainda podem estar relacionadas com a relação entre o número de diferentes elementos do sistema e o número de funções realizadas pelo sistema, de modo que um sistema com pequeno número de elementos diferentes e um maior número de funções seria mais complexo que um sistema com um grande número de elemento, mas com relativamente poucas funções. Desta forma, alguns sistemas podem ser mais ou menos complexos que outros. Mais tarde será discutida a relação entre complexidade de um sistema e seu grau de estabilidade frente a perturbações, noção importante na análise de qualquer tipo de sistema biológico. Particularmente em sistemas biológicos, padrões complexos correspondem à organização hierárquica do sistema e têm a potencialidade de se modificar em função das interações com o meio circunvizinho (Souza \& Manzatto 2000). Este tipo de sistema, com capacidade de responder de forma 
organizada aos estímulos externos, denomina-se Sistema Adaptativo Complexo (SAC) (Gell-Mann 1996).

Sistemas biológicos, de árvores a comunidades florestais, possuem um padrão típico de estruturação com propriedades auto-similares que independem da escala de observação. Por exemplo, o padrão de ramificação de uma árvore formando sua copa pode ser igualmente observado no padrão de ramificação das nervuras de uma única folha. Este padrão auto-repetitivo de construção de estruturas tem sido associado à construção de estruturas fractais. Fractais são conjuntos cuja forma é extremamente irregular ou fragmentada e que apresentam essencialmente o mesmo padrão em todas as escalas. A geometria dos fractais permite uma estimativa muito mais precisa da dimensão de objetos naturais, que são muito distintos das formas euclidianas, possuindo muitas aplicações em biologia. De uma forma geral, a dimensão fractal mede a ocupação irregular de um espaço devido a um processo de crescimento (Vicsek 2001). Por exemplo, colônias de bactérias crescendo em um meio de cultura homogêneo e rico em nutrientes tendem a formar estruturas esféricas (ocupação homogênea de espaço). Porém, se o meio apresentar recursos limitados e/ou alta densidade, simulando ambientes naturais, as colônias de bactérias apresentarão uma forma irregular (fractal) caracterizando um processo não-homogêneo de ocupação de espaços (figura 1).

Sistemas biológicos não são apenas estruturalmente, mas também funcionalmente complexos. Plantas ou comunidades vegetais são sistemas que evoluem no tempo, isto é, seus estados, em todas as escalas de organização, sofrem modificações em uma determinada faixa de amplitude em função do estado anterior do sistema. Tais modificações imprimem uma dinâmica temporal que raramente pode ser caraterizada por alterações proporcionais no sistema, ou seja, as dinâmicas temporais de sistemas biológicos são predominantemente não-lineares. Tais sistemas com dinâmicas não-lineares podem ser particularmente sensíveis a perturbações externas, de modo que a previsibilidade de seus comportamentos futuros torna-se muito difícil. Este tipo de dinâmica pode ser caracterizada como uma dinâmica caótica. Portanto o termo caos ou caótico refere-se ao tipo de sistema cujos processos, como, por exemplo, o metabolismo respiratório ou o processo sucessional em uma floresta, têm dinâmicas temporais sensíveis a variações externas, e previsões de longo alcance tornam-se cada vez mais improváveis. Todavia, este tipo de comportamento não deve ser confundido com um comportamento puramente
A

B
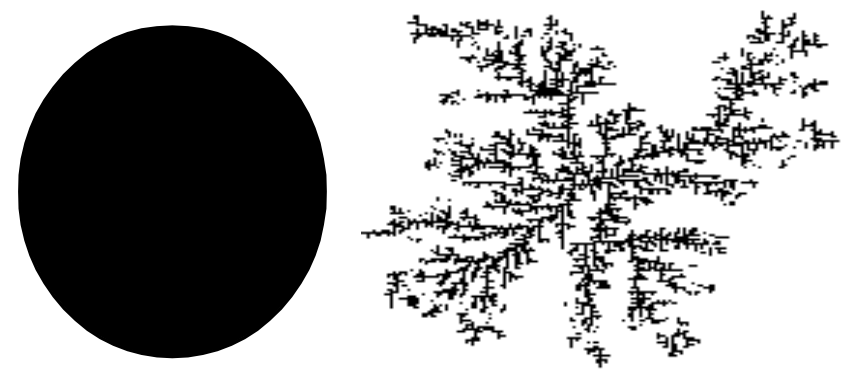

Figura 1. Exemplos de ocupação de um espaço de forma homogênea (A), e de forma não homogênea (B). Simulação da figura (B) realizada com o modelo de construção de objetos fractais tipo DLA(Diffusion-limited Aggregation) introduzido por Witten e Sander (1983).

Figure 1. Examples of homogenous (A) and a heterogeneous (B) space occupation. The simulation in figure (B) was performed with a model for construction of fractal objects of the DLA (Diffusion-limited Aggregation) type, introduced by Witen and Sander (1983).

probabilístico ou aleatório. Sistemas dinâmicos caóticos estão associados, pelo menos teoricamente, a equações matemáticas que descrevem sua dinâmica no espaço de estados (espaço cartesiano de $\mathrm{N}$ dimensões, onde cada dimensão está relacionada a uma variável observada no sistema). Portanto, são sistemas que, embora imprevisíveis em longo prazo, possuem um determinismo subjacente que permite sua modelagem por meio de equações diferenciais. De fato, sistemas caóticos são caracterizados por atratores, que são regiões restritas do espaço de estados para onde as trajetórias do sistema convergem. No caso de um sistema com dinâmica puramente aleatória, o atrator do sistema preenche o espaço de estados inteiramente, isto é, não há nenhum tipo de organização temporal, a dinâmica do sistema é totalmente imprevisível a curto ou longo prazo, enquanto que em um sistema caótico sua dinâmica fica restrita a uma região do espaço de estados (o atrator).

A seguir, trataremos de alguns exemplos de aplicações dessas teorias em diversas áreas da pesquisa biológica, em especial à Botânica, com o intuito de proporcionar uma visão com perspectiva prática e ganhos adicionais de informação e conhecimento sobre os processos e estruturas biológicas.

\section{A emergência da complexidade}

Montar uma proteína não é nada fácil. As relações de energia e logística celular envolvidos na montagem 
de uma molécula protéica é provavelmente mais complexa do que a mais sofisticada tecnologia já desenvolvida pelo homem. Mesmo assim, a síntese de proteínas ocorre todos os dias em milhões de células de uma única planta. Mais do que montá-las, é necessário controlar seus tempos de vida e destruí-las no momento certo, para permitir que o sistema continue funcionando. A proteína é um exemplo que pode ser extrapolado para todas as outras moléculas orgânicas (carboidratos, lipídeos, compostos do metabolismo secundário e ácidos nucléicos, por exemplo). Pode-se imaginar, assim, que um sistema extremamente complexo, como uma célula vegetal, esteja se renovando a todo momento e que, para isto, é vital que a repetição contenha pouco ou nenhum erro. Uma pergunta fundamental seria: como pode tudo isso acontecer em paralelo e, mesmo assim, repetir praticamente a mesma coisa no dia seguinte? Tal observação poderia ser criticada através da constatação de que na realidade, a planta de amanhã não é exatamente a mesma de hoje, pois amanhã já terá ocorrido desenvolvimento. Porém, tal crítica é uma forma de transpor o problema para um outro nível de complexidade, pois o desenvolvimento também ocorre sempre através de uma seqüência muito similar de eventos (ou mecanismos). Através de mecanismos de reiteração de processos, talvez com maior propensão a erros, o sistema acaba produzindo células, órgãos e organismos bastante similares.

De maneira mais geral, podemos conceber o desenvolvimento de sistemas organizados, ou o fenômeno da auto-organização, como um processo que induz variações na complexidade de um sistema, freqüentemente aumentos de complexidade, simultaneamente estrutural e funcional, resultante de uma sucessão de desorganizações geradas por distúrbios internos ou externos ao sistema Assim, tais desorganizações provocadas pelo ambiente externo, sujeitas à segunda lei da termodinâmica (o aumento da entropia no sistema), constituem um ruído que afeta o desenvolvimento do sistema. O ruído provoca erros no sistema, que a posteriori podem ser responsáveis pelo aumento de variedade (complexidade) no sistema (Atlan 1986), por exemplo, aumentando as taxas de mutação de uma espécie, conferindo-lhe maior grau de adaptação e, portanto, aumentando suas chances de sobrevivência em um ambiente variável (Schulze \& Mori 1993).

Outra importante propriedade intrínseca de sistemas auto-organizados é a emergência. A teoria da emergência diz que a totalidade do sistema é maior que a soma de suas partes e o todo exibe padrões e estruturas que surgem espontaneamente das partes. $\mathrm{O}$ crescimento de um sistema auto-organizado é autônomo, isto é, sem controladores externos, possibilitando o surgimento de novidades imprevisíveis (Ayres 1994). Entretanto, é possível fazer distinções significativas observando certos aspectos do sistema como um todo. Um dos aspectos mais importantes é a hierarquia ascendente de ordem. Em sistemas de vários níveis (moléculas-células-tecidosórgãos), a hierarquia tem especial importância; cada nível inclui todos os níveis inferiores criando uma rede organizada auto-controlada (Jantsch 1992, Souza \& Manzatto 2000).

De acordo com a visão sistêmica, as propriedades essenciais de um organismo vivo são propriedades do todo, que nenhuma das partes possui. Elas surgem das interações e das relações entre as partes, e desaparecem quando o sistema é dissecado em suas partes isoladas (Bertalanffy 1968, Holland 1995, Capra 1997).

Segundo Bresciani \& D'Ottaviano (2000), para poder tratar um problema sob a perspectiva de um enfoque sistêmico, algumas condições e princípios devem ser reconhecidos. Entre eles podemos destacar os seguintes: a) existência do sistema com uma estrutura subjacente, constituída por um conjunto de elementos e pelas relações entre eles (hierarquia), e com uma funcionalidade, b) caracterização de elementos internos, externos e de fronteira, c) possibilidade de o sistema receber energia, matéria e informação do ambiente, transformá-las internamente e transmiti-las para o exterior, d) possibilidade de manutenção do equilíbrio estrutural e funcional do sistema em sua relação com o ambiente externo, e) possibilidade de mudança de estado com a emergência de novo estado, que caracteriza a criação ou a evolução, através do mecanismo de adaptação estrutural e funcional.

\section{A morfologia fractal das plantas}

Objetos com características fractais possuem autosimilaridade em sua estrutura, e são gerados por processos de crescimento que proporcionam uma ocupação não-homogênea do espaço. Isto possibilita a utilização de modelos baseados na geometria fractal para a simulação do padrão de crescimento de organismos que possuem crescimento modular, como as plantas. Estudos têm sido desenvolvidos utilizando medidas de dimensão fractal, isto é, medidas fracionárias da dimensão geométrica de um objeto qualquer, para estudar a complexidade da forma de folhas possibilitando a discriminação de espécies por computador associadas a outros tipos de medidas morfométricas (Borkwoski 
1999) e podendo ser útil para a identificação de hídridos (Vlcek \& Cheung 1985). A dimensão fractal também pode ser empregada como uma medida quantitativa para o estudo do crescimento e desenvolvimento de plantas caracterizando mudanças de complexidade estrutural (Corbit \& Garbary 1995, Alados et al. 1999).

De forma geral, a dimensão fractal é um indicador direto da maior complexidade da forma de um determinado objeto: quanto maior a dimensão fractal, maior o nível de complexidade da forma avaliada. Assim, a dimensão fractal permite avaliar e comparar padrões de ramificação de copas de árvores (Berger 1991, Long 1994) e especialmente de sistemas radiculares (Tatsumi et al. 1989, Nielsen et al. 1997a). Como avaliação de complexidade, a dimensão fractal pode, então, ser utilizada como uma medida quantitativa em estudos que envolvam experimentos com estresses ambientais que possam alterar a forma das plantas.

Uma forma de estudar o crescimento vegetal que envolve a geometria fractal constitui os Sistemas de Lindenmayer (L-Systems). Através da construção desses modelos, que envolvem o conceito básico de autosimilaridade, é possível simular, em computador, uma variedade quase infinita de formas geométricas que simulam surpreendentemente bem as formas vegetais (Prusinkiewics \& Lindenmayer 1990). Este tipo de modelo tem sido utilizado para simular a formação de árvores, inclusive com a utilização de desvios de simetria simulando o crescimento em um ambiente natural (Kruszewski \& Whitesides 1998). Fournier e Andrieu (1998) utilizaram um L-System associado a modelos biofísicos para simular um modelo tri-dimensional do desenvolvimento de plantas de milho em diferentes condições microclimáticas.

\section{Complexidade em fisiologia e bioquímica de plantas: caminhos para o caos}

Sistemas biológicos, do nível molecular ao ecológico, são freqüentemente controlados por mecanismos nãolineares de retroalimentação, isto é, possuem laços recorrentes (feedback) que permitem a amplificação de sinais de comunicação pelo sistema (feedback positivo), bem como propicia uma regulação do sistema através de relações de antagonismo (feedback negativo). Assim, não seria surpreendente que esses mecanismos mostrassem comportamentos irregulares, como os caóticos (May 1989, Mosekild \& Mosekild 1991). Recentemente, Hütt \& Lüttge (2002) publicaram uma revisão sobre modelagens não-lineares em fisiologia de plantas. Os autores destacam a importância de representações matemáticas não-lineares para uma melhor compreensão de um mundo vivo em que certas propriedades (emergentes) demandam especial instrumental analítico baseado nas teorias sobre complexidade.

De fato, embora a literatura ainda seja muito escassa, alguns autores têm observado a ocorrência de dinâmica caótica em diferentes processos fisiológicos de plantas. Lüttge \& Beck (1992) mostraram a possível existência de dinâmica caótica no ritmo circadiano em espécies com metabolismo ácido crassuláceo (MAC). Krempasky et al. (1993) mostraram que, alterando a intensidade luminosa, podem surgir padrões temporais no comportamento do sistema fotossintético variando de periódico a caótico. Shabala et al. (1997) observaram a ocorrência de períodos duplos e triplos em correntes bio-elétricas em folhas de algumas espécies de plantas em resposta à temperatura.

Essas observações trazem a seguinte indagação. Por quê estas dinâmicas existem? Há algum tipo de "vantagem" adaptativa nestes tipos de comportamentos?

Segundo Møller \& Swaddle (1997), a dinâmica não linear dos fatores ambientais, assim como do metabolismo, torna improvável que uma estabilidade global de um sistema possa ser atingida. Em outras palavras, é improvável que todos os níveis de organização do sistema possam ser simultaneamente estáveis. Assim, parece provável que em um ambiente natural, onde sempre existem oscilações, plantas e outros organismos apresentem um metabolismo com dinâmica irregular, propiciando condições favoráveis de crescimento e adaptação. Estes são os SAC, Sistemas Adaptativo Complexos, (Gell-Mann 1996) que possibilitam o reconhecimento de regularidades no meio, condensando-as em "esquemas" ou modelos. Em cada caso, há vários modelos possíveis competindo e os resultados da ação sobre o ambiente retro-alimentam cada modelo e influenciam na competição entre eles. Shabala et al. (1997) consideram difícil acreditar que para plantas crescendo em um ambiente com flutuações naturais, respostas caóticas representem um comportamento anormal ou prejudicial, uma vez que elas têm sobrevivido durante milhões de anos de evolução em um ambiente em constante modificação. Dinâmicas caóticas permitem que o organismo tenha flexibilidade nas respostas às mudanças ambientais. Isto se deve ao fato de atratores caóticos coexistirem com um grande número de órbitas periódicas instáveis. Quando um sistema em um estado caótico é perturbado, ele pode responder ou permanecendo caótico ou estabelecendo sua dinâmica em uma das órbitas periódicas instáveis 
na vizinhança do atrator caótico (Møller et al. 1998). As órbitas periódicas instáveis são frequiências regulares na dinâmica do sistema, representando uma periodicidade que pode ser facilmente alterada por alguma perturbação.

Igualmente em sistemas enzimáticos comportamentos caóticos têm sido cada vez mais observados (Olsen \& Degn 1977, Nielsen et al. 1997b, Møller et al. 1998).

Recentemente Souza et al. (2004a), comparando a dinâmica temporal de trocas gasosas $\left(\mathrm{CO}_{2} \mathrm{e}_{2} \mathrm{O}\right)$ de plantas de girassol e beterraba submetidas à deficiência hídrica, verificaram uma maior capacidade de recuperação (homeostase) das plantas que apresentaram maior complexidade de suas dinâmicas. Isto suporta as hipóteses consideradas acima. Marcos Buckeridge e Ana Maria Baroni (resulados inéditos) verificaram que as respostas de folhas da bromélia Alcantarea imperialis V.L. Harms apresentam resposta de assimilação de $\mathrm{CO}_{2}$ na forma de um atrator não linear (figura 2). Este padrão de comportamento provavelmente sincroniza a resposta das folhas à luz com o padrão de variação natural sob o dossel da floresta. As folhas desta planta são capazes de responder à luz em forma de "sunfleck" (flashes de luz de curta duração que ocorrem sob o dossel nas florestas), o que provavelmente permite maior eficiência de uso da energia luminosa em ambientes com variação não linear na intensidade luminosa.

\section{O surgimento e evolução de florestas como o resultado da emergência da interação de indivíduos: as florestas também se comportam como redes}

SACs freqüentemente desenvolvem propriedades emergentes. Tais propriedades surgem em função das interações retro-alimentadoras locais entre os elementos

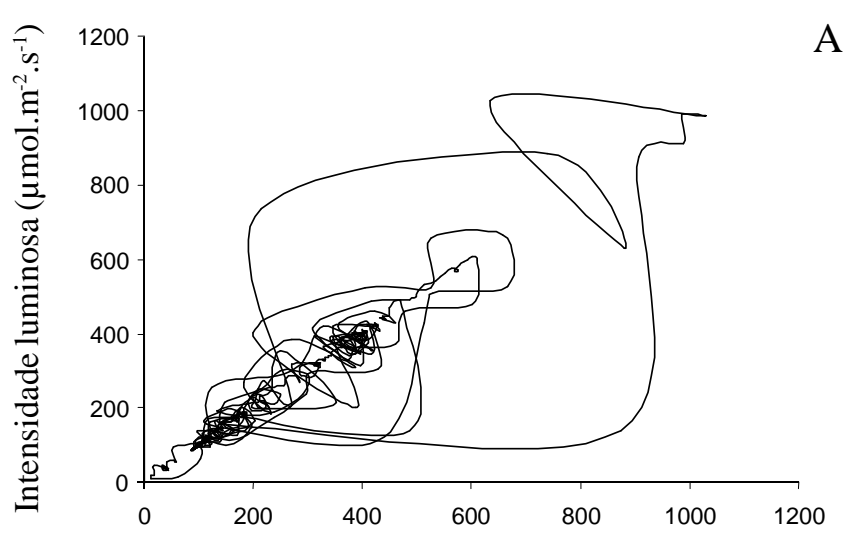

Intensidade luminosa $\left(\mu \mathrm{mol} . \mathrm{m}^{-2} \cdot \mathrm{s}^{-1}\right)$
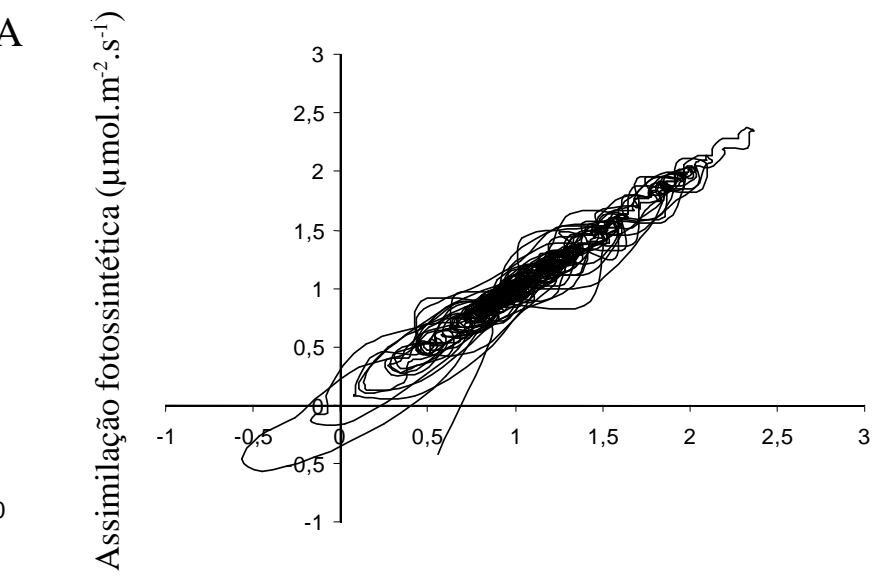

Assimilação fotossintética $\left(\mu \mathrm{mol} \cdot \mathrm{m}^{-2} \cdot \mathrm{s}^{-1}\right)$

Figura 2. Atratores formados pelas variações de (A) intensidade luminosa incidente sobre uma folha da bromélia Alcantarea imperialis V.L. Harms formado pela passagem de raios solares através do dossel e (B) respostas de assimilação de $\mathrm{CO}_{2}$ pela folha neste mesmo período. Os gráficos foram construídos através da coleta de dados (em um aparelho IRGA - Infra Red Gas Analyzer Licor 6400) de PAR (Photosynthetically Active Radiation) and A (Assimilation) correspondentes a 1192 pontos, sendo a periodicidade de 1 ponto por segundo. A construção dos atratores foi feita através da plotagem da sequiência de pontos contra ela mesma com defasagem de um ponto. Desta forma se obtém gráficos da trajetória do fenômeno ao longo do tempo. Estes dados também foram analisados com o programa Chaos Data Analyzer da Physics Academic Software, gerando as mesmas figuras (dados não publicados).

Figure 2. Atractors formed by (A) flecks of light reaching the surface of a leaf of the bromeliad Alcantarea imperialis V.L. Harms after interference tree branches and (B) $\mathrm{CO}_{2}$ assimilation by the leaf in the same period. The graphs were constructed after collection of data using and Infra Red Gas Analyser (Licor 6400). The number of points collected for Photosynthetic Active Radiation and $\mathrm{CO}_{2}$ assimilation were 1192 at a rate of 1 point per second. The construction of the attractor was performed by plotting each data sequence against itself with a delay of one second. In this plot, the "trajectory" of the phenomenon can be visualised. These data were also analysed using the software Chaos Data Analyser from Physics Academic Software (data not published). 
vizinhos de um sistema, possibilitando o surgimento de um padrão global de organização. Este tipo de processo organizacional é conhecido como processo "bottom-up" (de baixo para cima), em oposição aos processos do tipo "top-down" (de cima para baixo), nos quais a organização do sistema é imposta por algum tipo de agente controlador de escala superior, como, por exemplo, uma pressão seletiva muito forte em um ecossistema, ou a coordenação do cérebro sobre os movimentos do corpo. Todavia, é bastante provável que em um sistema biológico, ambos os processos, variando sua importância relativa, atuem sobre a organização do sistema durante sua evolução de longo prazo. Um exemplo de processo organizador do tipo "bottom-up" é o da organização de uma comunidade vegetal. As características relacionadas à distribuição ou frequiência de espécies e até mesmo a dinâmica de uma sucessão florestal, dependem dos estados fisiológicos de cada indivíduo em cada espécie frente às eventuais pressões de seleção natural. Assim, dependendo da capacidade dos organismos em responder às perturbações ambientais, a estrutura da comunidade em uma determinada região poderá variar ao longo do tempo.

A dinâmica da sucessão ecológica também pode ser abordada como um fenômeno de organização emergente (bottom-up). As formações de florestas tropicais têm sido caracterizadas como um mosaico sucessional com diferentes fases estruturais e florísticas induzidas pela abertura de clareiras no dossel. Sua dinâmica e manutenção decorrem dos diferentes ambientes gerados desse mosaico, nos quais as espécies possuem capacidade diferencial de sobreviver e se desenvolver (Pickett et al. 1987, Luken 1990, Solè \& Manrubia 1995).

Nessa complexa matriz dinâmica de micro-sítios, a disponibilidade de luz varia mais dramaticamente que qualquer outro recurso, influenciando criticamente o estabelecimento, crescimento, sobrevivência e reprodução das espécies vegetais (Chazdon et al. 1996, Strauss-Debenedetti \& Bazzaz 1996). Dessa forma, a utilização fotossintética da luz é um componente fundamental para a distribuição das espécies ao longo de um gradiente de regeneração de clareiras. Com base na sua capacidade de ocupação espacial e temporal das clareiras, as espécies podem ser divididas em dois grupos sucessionais: espécies sucessionais iniciais, que demandam luz, e espécies sucessionais tardias, que são tolerantes ao sombreamento. Em geral, as espécies do início da sucessão apresentam uma maior flexibilidade nas respostas em relação ao aumento de luminosidade (Chazdon et al. 1996, Strauss-Debenedetti \& Bazzaz
1996).

Segundo Solè \& Manrubia (1995) e Solè et al. (1999), a dinâmica de clareiras em uma floresta tropical pode estar relacionada a um fenômeno comum na natureza conhecido como "criticalidade autoorganizada". Sistemas criticamente auto-organizados, ou no estado crítico, são aqueles sistemas onde todos os elementos constituintes do sistema podem influenciar uns aos outros, direta ou indiretamente. Desta forma, algumas perturbações, independentes de sua escala, podem influenciar o sistema com um todo (Bak \& Chen 1991, Jensen 1998).

Deste exemplo pode-se perceber que o padrão global da dinâmica de clareiras emerge das diferentes capacidades dos indivíduos de cada espécie em lidar com os recursos disponíveis. As interações espaçotemporais entre os diferentes indivíduos em um dado ambiente estabelecem uma organização em rede, que apresenta certas características que só podem ser observadas em uma escala ecológica. Como foi discutido anteriormente, tais interações são tipicamente não lineares o que confere ao sistema uma certa estabilidade em relação a perturbações ambientais. Tal estabilidade é mantida pela existência de vários atratores possíveis no sistema, que podem ser alcançados devido à plasticidade/flexibilidade dos indivíduos que formam a comunidade.

$\mathrm{O}$ aumento de complexidade em um ecossistema pode então ser relacionado a um aumento de estabilidade (Van Voris \& O'Neill 1980, Hastings et al. 1993, McCann 2000). Particularmente em ecologia, o debate diversidade-estabilidade possui uma importância marcante no contexto das discussões sobre o impacto das atividades humanas sobre a manutenção da biodiversidade e para os programas de preservação ou recuperação de comunidades (MacCann 2000). Diversidade, que considera o número de diferentes espécies que compõe um ecossistema, pode assim ser facilmente relacionada a uma das medidas de complexidade do ecossistema (a outra está relacionada às interações entre as espécies formando redes, por exemplo, como as teias ou cadeias alimentares). De uma forma geral, o aumento da diversidade, em média, pode levar a um aumento da estabilidade do ecossistema. Esta relação positiva entre diversidade e estabilidade pode ser explicada de duas formas. Uma é que aumentando a diversidade aumentam-se as chances que pelo menos algumas espécies respondam diferentemente a condições ambientais variáveis ou perturbações. A segunda explicação é que, quanto maior for a diversidade, maiores as chances de um ecossistema 
possuir redundância funcional contendo espécies que são capazes de substituir funcionalmente espécies importantes. Isto significa que quanto maior o número de espécies, maior será o número de relações fracas entre os componentes do ecossistema, diminuindo a importância relativa de interações fortes que podem desestabilizar o sistema caso sejam significativamente perturbadas (MacCann 2000).

Por outro lado, em uma escala global, compreendendo a biosfera como um todo, esses mesmos princípios de organização emergente podem ser utilizados para explicar e modelar o surgimento de diferentes biomas como sendo diferentes atratores representando diferentes sistemas surgindo sob diferentes condições ambientais. Uma vez que a própria vida e os mais variados sistemas vivos (células, organismos, comunidades) são sistemas dinâmicos não lineares, freqüentemente apresentando dinâmica caótica, determinadas diferenças ambientais poderiam levar à evolução de ecossistemas específicos, em um tempo relativamente curto. A importância de dinâmicas caóticas para a organização espaço-temporal de uma comunidade vegetal tem sido reconhecida (Hastings et al. 1993, Stone \& Ezrati 1996, Anand 1997). Da mesma forma, alguns autores (Shishkin 1992, Amzallag 1999) têm sugerido que a formação de ecótipos estáveis pode ser alcançada em poucas gerações com base em processos epigenéticos. Desta forma, cada ecossistema também poderia ser encarado como um atrator em um espaço de possibilidades que depende de condições ambientais específicas.

Este tipo de evolução espaço-temporal pode ser mais eficientemente modelado por uma classe relativamente nova de modelos conhecidos como autômatos celulares (ACs). O objetivo geral deste tipo de modelagem é compreender o comportamento coletivo de vários elementos simples com propriedades semelhantes interagindo em um espaço de possibilidades gerando comportamentos complexos (Wolfram 1984, Hütt \& Lüttge 2002). Os ACs, introduzidos na década de 1960 pelo matemático John von Neumann, são sistemas definidos em uma rede de células (um tabuleiro de xadrez, por exemplo, é uma rede bidimensional), onde cada célula contendo um valor numérico evolui no tempo, segundo um conjunto de regras pré-determinadas, interagindo com as células vizinhas. A cada passo da evolução do sistema, os valores de todas as células são atualizados simultaneamente. A figura 3 mostra a evolução de um AC hipotético para exemplificar seu funcionamento. A figura 4 ilustra um padrão fractal gerado por um outro tipo de AC, mostrando o potencial deste modelo para simular formas geométricas

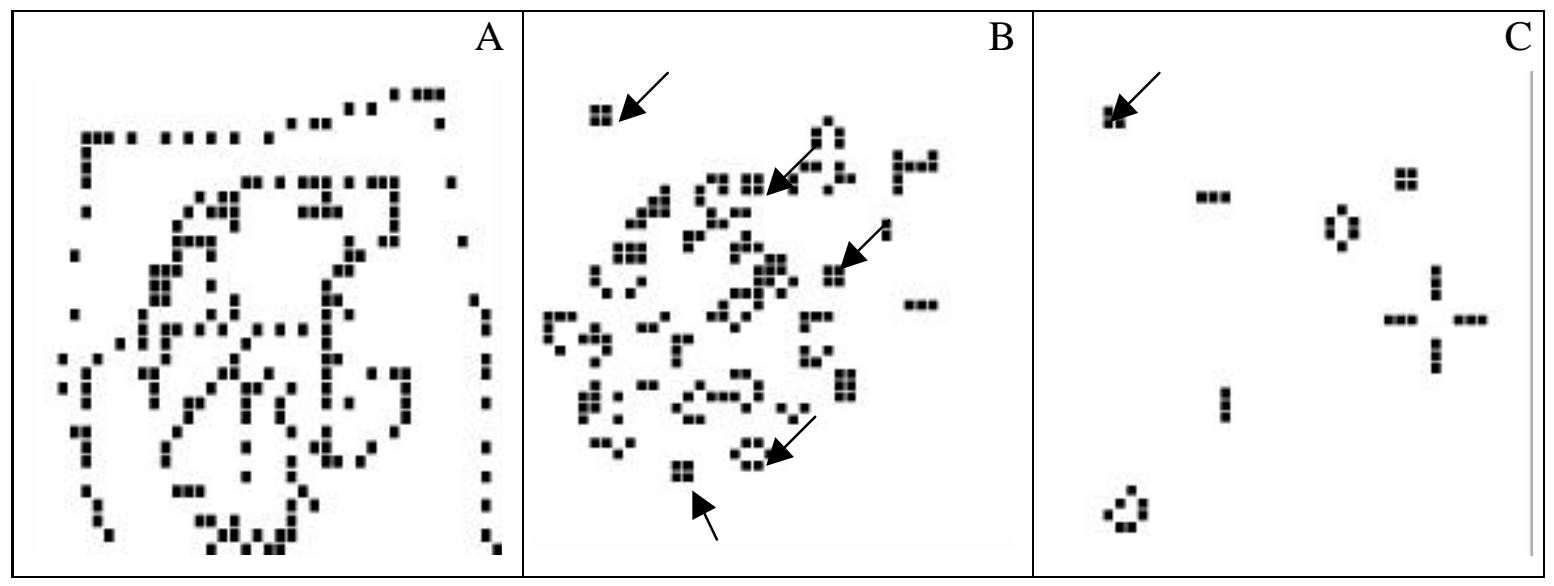

Figura 3. Representação do funcionamento de um autômato celular gerado pelo programa Life-3000. (A) início da simulação com a disposição aleatória de uma população formada por 210 células escuras; (B) estado da população após um tempo de 10 gerações (t10). Neste novo estado a população inicial foi reduzida para 166 células escuras e já podem ser observadas algumas estruturas estáveis (indicadas pelas setas) não previstas pelas regras de aplicação do modelo; (C) Após 115 gerações o sistema alcançou seu estado estacionário, com uma população de 39 células. A seta indica uma estrutura estável formada desde t10. Regras de funcionamento do AC: 1) para uma célula branca tornar-se escura é necessário ter exatamente 3 vizinhos adjacentes escuros; 2) para uma célula escura continuar escura na próxima geração precisa ter pelo menos 2 vizinhos escuros e no máximo 3 vizinhos escuros. Este simples exemplo ilustra a capacidade de sistemas compostos por elementos similares gerarem padrões estáveis imprevisíveis a partir de regras de interação simples. 


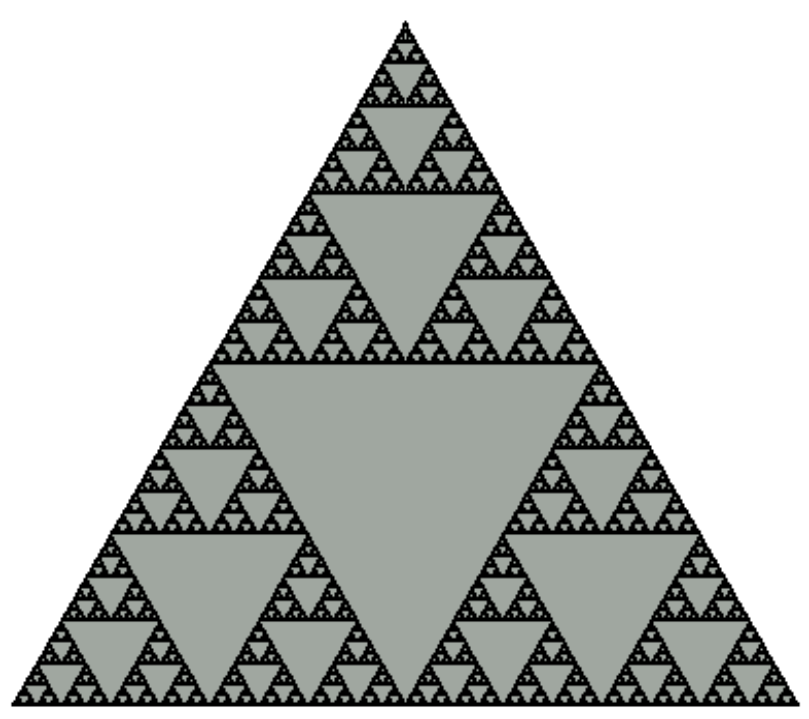

Figura 4. Exemplo de uma estrutura fractal (triângulo de Sierpinsky) gerada por um modelo de autômato celular.

Figure 4. Example of a fractal structure (The Sierpinsky triangle) generated by a cellular automaton.

complexas. O conjunto de regras de cada modelo depende do fenômeno a ser modelado. Assim, por exemplo, poderíamos considerar os estômatos distribuídos em uma folha como um tipo de autômato celular, no qual as regras de interação entre as células vizinhas dependem de gradientes de água estabelecendo fluxos de entrada e saída de água das células estomáticas, promovendo sua abertura ou fechamento. Naturalmente, como as regras dos ACs são definidas por um programador externo, ACs não são, em si, sistemas auto-organizados, mas retêm certas características de sistemas auto-organizados, uma vez que freqüentemente simulam comportamentos emergentes não programados, similares aos dos fenômenos biológicos modelados. ACs têm sido usados para modelar e simular o comportamento da dinâmica de populações, pequenas comunidades ou mesmo os efeitos ambientais como a luz ou fogo sobre a dinâmica espaço-temporal de florestas (Grist 1999, Lett et al. 1999).

\section{Aspectos da complexidade de sistemas em rede}

Basicamente, sistemas biológicos são organizados em padrões de redes de conexões entre os elementos constituintes. Assim, o funcionamento do sistema como um todo ou de partes do sistema (subsistemas) depende da modulação destas redes.

Uma das formas utilizadas para modelar o funcionamento de redes em fenômenos biológicos é através das Redes Neurais Artificiais (RNAs). As RNAs foram desenvolvidas com base no funcionamento de redes de neurônios reais. Apesar de procedimentos de RNAs terem sido aplicados em engenharia e para modelar o funcionamento de mecanismos em biologia animal (Lau et al. 2002), pouco foi feito para ciências vegetais até o momento. Uma RNA é um programa de computador que aprende a prever o resultado de uma combinação de variáveis com base em variações passadas destas variáveis. Uma explicação simplificada do funcionamento de uma RNA seria que esta dá pesos diferentes às variáveis e através da valorização ou não de sua contribuição para um determinado resultado. Combinações que apresentam os menores erros entre os resultados previstos e os reais são mantidas. As plantas podem ser submetidas à análises de sua atuação na forma de rede usando RNAs. Recentemente, M.B.P. de la Vega, M. Saraiva \& M.S. Buckeridge (dados não publicados) construíram uma rede neural artificial com dados ambientais relacionados às respostas fotossintéticas do jatobá. O treinamento da rede possibilitou uma previsão do potencial fotossintético de uma planta jovem de jatobá com apenas $10 \%$ de erro, mostrando não somente que a planta responde às variáveis ambientais em forma de rede, mas que as RNAs poderiam constituir uma aplicação prática concreta de utilização de dados do ambiente para fazer previsões com erro relativamente baixo sobre a fisiologia da planta.

Em geral, na pesquisa Botânica, utilizamos determinados parâmetros para avaliar ou descrever os

Figure 3. Representation of the functioning mechanism of a cellular automaton. The software Life-3000 was used. (A) simulation at the beginning showing a random distribution formed by 210 dark cells; (B) population state after 10 generations ( $\mathrm{t} 10)$. In this new state, the initial population has been reduced to 166 dark cells and some stable structures (arrows) can be seen. These have not been forecasted by the model; (C) After 115 generations, the system reached a steady state, with a population of 39 cells. The arrows indicate a stable structure since t10. Rules of the cellular automaton: 1) for a white cell to turn dark it is necessary that the 3 adjacent neighbours are dark; 2) for a dark cell to continue dark, the next generation needs at least 2 dark neighbours and at the maximum 3 dark neighbours. This simple example illustrates the capacity of systems composed of similar elements to generate stable patterns which are impossible to forecast from the sole integration of the rules. 
estudos de um determinado sistema (do metabolismo às comunidades) e normalmente nos atemos a valores médios desses parâmetros para caracterizar o sistema em estudo, analisando propriedades estatísticas das medidas. Entretanto, qualquer parâmetro que medimos está necessariamente associado a algum tipo de rede e é modulado por controladores de fluxo, em um dado sistema. Assim, a inclusão desses parâmetros em uma rede é uma ferramenta útil para acessar aspectos da complexidade do sistema.

Amzallag (2001a) propõe um método relativamente simples de acessarmos a ocorrência de diferenças de modulação (controle) de um sistema em estudo a partir de reinterpretações de análises estatísticas dos parâmetros medidos. Segundo este autor, é geralmente aceito que variações ao redor da média não têm significado biológico. Todavia, já existem fortes indícios de que o nível de ruído (variabilidade no conjunto de dados) possa ter um significado biológico (Trewavas 1986, Green 1996, Erdei et al. 1998, Amzallag 1999, 2001b, Hütt \& Lüttge 2002). Desta forma, segundo Amzallag (2001a), a falta de diferença significativa na média de um valor $\mathrm{X}$ afetado por um fator $\mathrm{Y}$, não significa que $X$ não tenha sofrido modificação alguma. Quando um teste de comparação de médias indica diferença significativa no valor médio de um parâmetro $\mathrm{X}$ afetado por uma variável $\mathrm{Y}$, podemos dizer que $\mathrm{Y}$ afeta $X$ linearmente, como em uma relação determinística de causa-efeito. Todavia, quando o resultado não é significativo podemos inferir que o parâmetro X pode estar sendo modulado por uma rede com diversos níveis de redundância (elementos estruturalmente similares que realizam uma mesma função) ou degeneração (diferentes vias para a realização de uma mesma função). Redes com maior ou menor grau de redundância ou degeneração, possibilitam a manutenção da homeostase dos sistemas biológicos quando perturbados por fatores externos (Trewavas 1986, Edelman \& Gally 2001). Como conseqüência, o nível de variabilidade em um parâmetro $\mathrm{X}$ medido pode servir como uma estimativa do nível de redundância ou degeneração em sua regulação. Mudanças no nível de variabilidade em um dado parâmetro podem ser analisadas independentemente de mudanças no valor médio do parâmetro quando a variabilidade é normalizada como um coeficiente de variação $\mathrm{CV}(\mathrm{y})=100 \mathrm{DP}(\mathrm{y}) / \mathrm{M}(\mathrm{y})$, onde $\mathrm{PD}$ é o desvio padrão da média M (Amzallag 2001a).

A comparação de valores de CV pode indicar alterações na rede do sistema em estudo, mas não fornece informações sobre a natureza destas mudanças.
Assim, Amzallag (2001a) sugere a avaliação da conectância da rede através da análise dos coeficientes de correlação $r$ normalizados: $z=0,5 \ln [(1+r) /(1-r)]$. Um coeficiente de correlação pode não ser considerado apenas para testar a significância de uma correlação, mas também como uma medida da força da relação (conexão) entre dois parâmetros. Assim, alterações dos padrões de conexão entre os parâmetros da rede podem sinalizar diferentes vias de controle induzidas em respostas a estímulos externos ou por processos de desenvolvimento endógenos.

Alguns trabalhos recentes (Souza et al. 2004b, Prado et al. 2004) vêm utilizando a técnica de análise de redes para detectar variações sistêmicas de parâmetros fisiológicos de plantas submetidas a algum tipo de perturbação ambiental. Souza et al. (2004b), estudando o comportamento de espécies arbóreas submetidas à deficiência hídrica, verificaram que a conectância das redes de trocas gasosas das plantas, constituídas pelas relações entre condutância estomática, assimilação de $\mathrm{CO}_{2}$, transpiração e concentração intercelular de $\mathrm{CO}_{2}$, tendem a aumentar sob deficiência hídrica, sugerindo um maior controle sobre estes parâmetros em condições de restrição ambiental. Uma tendência similar também foi observada por Prado et al. (2004) analisando a rede constituída pelos parâmetros de trocas gasosas em uma comunidade de cerrado no período de seca. Como sugerido pela teoria (Trewavas 1986, Edelman \& Gally 2001), as alterações nas relações entre parâmetros em uma rede parecem ter um papel importante na estabilização do sistema, aumentando seu auto-controle (força auto-organizadora) quando perturbado por um estímulo ambiental.

\section{Conclusões e perspectivas}

As ferramentas representadas pelas descobertas no campo da aplicação da Matemática não linear à Biologia, mais especificamente à Botânica, possibilitam a interação de diferentes áreas desta última de uma forma ainda inédita.

Pode-se especular que os elementos utilizados pelos taxonomistas como base para o trabalho de classificação e identificação das plantas em diferentes grupos é um resultado da noção cada vez mais clara da existência de atratores que representam diferentes perspectivas de observação morfológica das plantas. A abordagem moderna de integrar dados morfológicos, químicos e moleculares vem trazendo uma visão nova na taxonomia, permitindo visualizar a evolução das espécies vegetais de uma maneira mais profunda. Tais perspectivas 
resultam da interação entre o genoma e o ambiente, que se relacionam com a capacidade intrínseca de resposta de células vegetais (um embrião ou um esporófito) aos estímulos ambientais (positivos ou negativos), de forma a desenvolver uma estrutura capaz de se estabelecer e se adaptar aos atratores correspondentes às variáveis ambientais vigentes enquanto aquele indivíduo estiver vivo. A planta integrada ao seu ambiente forma um conjunto que funciona em forma de rede através da qual os fluxos de informação e matéria da atmosfera ao genoma e vice-versa formam um equilíbrio dinâmico caracterizado por uma mescla de atratores periódicos, lineares e não lineares. Os indivíduos em si funcionam como redes com graus variáveis de conectividade em diferentes níveis de complexidade. Indivíduos da mesma espécie compreendem o elemento de redundância do sistema e as variações de forma e função estudadas pelos anatomistas constituem os diferentes graus de degeneração dos sistemas. A cada nível de complexidade, novas propriedades emergentes surgem, formando uma rede extremamente complexa que apresenta uma estabilidade notável e tenuamente equilibrada.

Há novas ferramentas para o anatomista avaliar aspectos da forma de células e tecidos nas plantas (p.ex.: dimensões fractais). Os fisiologistas, bioquímicos e biólogos moleculares já podem utilizar as abordagens de rede para compreender melhor como as diferentes células, tecidos e órgãos estão conectados em forma de expressão gênica diferencial, vias metabólicas, controle hormonal etc. Em um nível de complexidade ainda maior, os ecólogos vegetais têm atualmente novas ferramentas (p.ex.: autômatos celulares) para avaliar as inter-relações entre as plantas e seu ambiente biótico e abiótico. Aparentemente, no futuro próximo, será menos através do isolamento em compartimentos e mais de uma forma integrada que as abordagens multidisciplinares da Botânica possibilitarão uma nova visão, talvez simplesmente denominada de Biologia Vegetal ou mais precisamente Biologia dos Sistemas Vegetais.

Agradecimentos - Os autores agradecem as excelentes sugestões dos colegas Fernando Martins e Fábio Scarano.

\section{Referências bibliográficas}

ALADOS, C. L., ESCOS, J., EMLEN, J. M. \& FREEMAN, D. C. 1999. Characterization of branch complexity by fractal analyses. International Journal of Plant Science 160:147-155.
AMZALLAG, G.N. 1999. Plant evolution: toward an adaptive theory. In Plant responses to environmental stresses: from phytohormones to genome reorganization. (H.R. Lerner, ed.). M. Dekker, New York, p.171-245.

AMZALLAG, G. N. 2001a. Data analysis in plant physiology: are we missing the reality? Plant, Cell and Environment 24:881-890.

AMZALLAG, G. N. 2001b. Maturation of integrated functions during development. I Modifications of the regulatory network during transition periods in Sorghum bicolor. Plant, Cell and Environment 24:337-345.

ANAND, M. 1997. The fundamental nature of vegetation dynamics: a chaotic synthesis. Coenoses 12:55-62.

ATLAN, H. 1986. Entre le cristal et la fumée. Éditions du Seuil, Paris.

AYRES, R.U. 1994. Information, entropy, and progress: a new evolutionary paradigm. AIP Press, New York.

BAK, P. \& CHEN, K. 1991. Self-organized criticality. Scientific American January:26-33.

BERGER, D.S. 1991. Modification of a simple fractal tree growth scheme: implications on growth, variation, and evolution. Journal of Theoretical Biology 152:513-529.

BERTALANFFY, L.V. 1968. General System Theory. Braziller, New York.

BORKOWSKI, W. 1999. Fractal dimension based features are useful descriptors of leaf and shape. Canadian Journal of Forestry Research 29:1301-1310.

BRESCIANI, E.F. \& D'OTTAVIANO, I.M.L. 2000. Conceitos básicos de sistêmica. In Auto-organização: estudos interdisciplinares. (I.M.L. D’Ottaviano \& M.E.Q. Gonzales, eds.). v.30 CLE/UNICAMP, Campinas, p.283-306.

CAPRA, F. 1997. A teia da vida: uma nova compreensão científica dos sistemas vivos. Cultrix/Amana-Key, São Paulo.

CHAZDON, R.L., PEARCY, R.W., LEE, D.W. \& FETCHER, N. 1996. Photosynthetic responses of tropical forest plants to contrasting light environments. In Tropical forest plant ecophysiology (S.S. Mulkey, R.L. Chazdon \& A.P. Smith, eds.). Chapman \& Hall, New York, p.5-55.

CORBIT, J.D. \& GARBARY, D.J. 1995. Fractal dimension as a quantitative measure of complexity in plant development. Proceedings of the Royal Society of London B, 262:1-6.

EDELMAN, G.M. \& GALLY, J.A. 2001. Degeneracy and complexity in biological systems. Proceedings of the National Academy of Science 98:13763-13768.

ERDEI, L., SZEGLETS, Z., BARABÁS, K.N., PESTENÁCZ A., FÜLÖP, K., KALMÁR, L., KOVÁCS, A., TÓTH, B. \& DÉR, A. 1998. Environmental stress and the biological clock in plants: changes of rhythmic behaviour of carbohydrates, antioxidant enzymes and stomatal resistance by salinity. Journal of Plant Physiology 152:265-271.

FIEDLER-FERRARA, N. \& PRADO, C.C. 1994. Caos: uma introdução. Edgard Blücher, São Paulo. 
FOURNIER, C. \& ANDRIEU, B. 1998. A3D architectural and process-based model of maize development. Annals of Botany 81:233-250.

GELL-MANN, M. 1996. O quark e o jaguar. Rocco, Rio de Janeiro.

GREEN, P.B. 1996. Transductions to generate plant form and pattern: an essay on cause and effect. Annals of Botany 78:269-281.

GRIST, E.P.M. 1999. The significance of spatio-temporal neighbourhood on plant competition for light and space. Ecological Modelling 121:63-78.

HASTINGS, A., HOM, C.L., ELLNER, S., TURCHIN, P. \& GODFRAY, H.C.J. 1993. Chaos in ecology: is mother nature a strange attractor? Annual Review of Ecological Systems 24:1-33.

HOLLAND, J.H. 1995. Hidden order: how adaptation builds complexity, Reading, Mass: Addison-Wesley, London.

HÜTT, M.-TH. \& LÜTTGE, U. 2002. Nonlinear dynamics as a tool for modelling in plant physiology. Plant Biology 4:281-297.

JANTSCH, E. 1992. The self-organizing universe: scientific and human implications of the emerging paradigm of evolution. Pergamon Press, Oxford.

JENSEN, H.J. 1998. Self-organized criticality: emergent complex behavior in physical and biological systems. Cambridge University Press, New York.

KREMPASKY, J., SMRCINOVA, M. \& BALLO, P. 1993. Periodicity and chaos in a photosynthetic system. Photosynthesis Research 37:159-164.

KRUSZEWSKI, P. \& WHITESIDES, S. 1998. Ageneral random combinatorial model of botanical trees. Journal of Theoretical Biology 191:221-236.

LAU, B., STANLEY, G.B. \& DAN, Y. 2002. Computational subunits of visual cortical neurons revealed by artificial neural networks. Proceedings of the National Academy of Sciences of USA 99:8974-8979.

LETT, C., SILBER, C. \& BARRETS, N. 1999. Comparison of a cellular automata network and an individual-based model for simulation of forest dynamics. Ecological Modelling 121:277-293.

LONG, C.A. 1994. Leonardo da Vinci's rule and fractal complexity in dichotomous trees. Journal of Theoretical Biology 167:107-113.

LUKEN, J.O. 1990. Directing ecological succession. Chapman \& Hall, London.

LÜTTGE, U. \& BECK, F. 1992. Endogenous rhythms and chaos in crassulacean acid metabolism. Planta 188:28-38.

MANDELBROT, B.B. 1982. The fractal geometry of nature, W.H. Freeman and Co., New York.

MAY, R.M. 1976. Simple mathematical models with very complicated dynamics. Nature 261:459-467.

MAY, R.M. 1989. The chaotic rhythms of life. New Scientist 124: $21-25$.

MCANN, K.V. 2000. The diversity-stability debate. Nature 405:228-233.
MØLLER, A.P. \& SWADDLE, J.P. 1997. Asymmetry, Developmental Stability, and Evolution. Oxford University Press, Oxford.

MØLLER, A.C., HAUSER, M.J.B. \& OLSEN, L.F. 1998. Oscilations in peroxidase-catalyzed reactions and their potential function in vivo. Biophysical Chemistry 72: 63-72.

MOREIRA, I.C. 1999. Fractais. In Complexidade e Caos, (H.M. Nussenzveig, ed.). Editora UFRJ/COPEA, Rio de Janeiro, p. 51-82.

MOSEKILD, E. \& MOSEKILD, L. 1991. Complexity, chaos, and biological evolution. Plenum Press, Series B: Physics v.270, New York.

NIELSEN, K.L., LYNCH, J.P. \& WEIS, H.N. 1997a. Fractal geometry of bean root systems: correlations between spatial and fractal dimension. American Journal of Botany 84:26-33.

NIELSEN, K., SORENSEN, P.G. \& HYNNE, F. 1997b. Chaos in glycolysis. Journal of Theoretical Biology 186:303-306.

OLSEN, L.F. \& DEGN, H. 1977. Chaos in an enzyme reaction. Nature 267:177-178.

PICKETT, S.T.A., COLLINS, S.L. \&ARNESTO, J.J. 1987. A hierarchical consideration of causes and mechanisms of succession. Vegetatio 69:109-114.

PRADO, C.H.B.A., WENHUI, Z., ROJAS, M.H.C. \& SOUZA, G.M. 2004. Seasonal leaf gas exchange and water potential in a cerrado woody species community. Brazilian Journal of Plant Physiology 16:7-16.

PRUSINKIEWICZ, P. \& LINDENMAYER, A. 1990. The algorithmic beauty of plants. Springer-Verlag, New York.

RUELLE, D. 1989. Chaotic evolution and strange attractors. Cambridge University Press, Cambridge.

SCHULZE, G. \& MORI, S. 1993. Increases in environmental entropy demand evolution. Acta Biotheoretica 41:149-164.

SHABALA, S., DELBURGO, R. \& NEWMAN, I. 1997. Observations of bifurcation and chaos in plant physiological responses to light. Australian Journal of Plant Physiology 24:91-96.

SHISHKIN, M.A. 1992. Evolution as a maintenance of ontogenetic stability. Acta Zoologica Fennica 191:37-42.

SOLÈ, R.V. \& MARRUBIA, S.C. 1995. Are rainforests selforganized in a critical state? Journal of Theoretical Biology 173:31-40.

SOLÈ, R.V., MANRUBIA, S.C., BENTON, M., KAUFFMAN, S.A. \& BAK, P. 1999. Criticality and scaling in evolutionary ecology. Trends in Ecology and Evolution 14:156-160.

SOUZA, G.M. \& MANZATTO, A.G. 2000. Hierarquia autoorganizada em sistemas biológicos. In Auto-organização: estudos interdisciplinares. (I.M.L. D’Ottaviano \& M.E.Q. Gonzáles, eds.), v.30 CLE/UNICAMP, Campinas, p.153-173. 
SOUZA, G.M., OLIVEIRA, R.F. \& CARDOSO, V.J.M. 2004a. Temporal dynamics of stomatal conductance of plants under water deficit: can homeostasis be improved by more complex dynamics? Brazilian Archives of Biology and Technology 47(3) (no prelo).

SOUZA, G.M., RIBEIRO, R.V., SANTOS, M.G., RIBEIRO, H. L. \& OLIVEIRA, R.F. 2004b. Functional groups of forest succession as dissipative structures: an applied study. Brazilian Journal of Biology 64(4) (no prelo).

STONE, L. \& EZRATI, S. 1996. Chaos, cycles and spatiotemporal dynamics in plant ecology. Journal of Ecology 84:279-291.

STRAUSS-DEBENEDETTI, S. \& BAZZAZ, F.A. 1996. Photosynthetic characteristics of tropical trees along successional gradients. In Tropical forest plant ecophysiology (S.S. Mulkey, R.L. Chazdon \& A.P. Smith, eds.). Chapman \& Hall, New York, p.162-186.
TATSUMI, J., YAMAUCHI, A. \& KONO, Y. 1989. Fractal analysis of plant root systems. Annals of Botany 64:499-503.

TREWAVAS, A.J. 1986. Understanding the control of plant development and the role of growth substances. Australian Journal of Plant Physiology 13:447-457.

VAN VORIS, P. \& O'NEILL, R.V. 1980. Functional complexity and ecosystem stability. Ecology 61:1352-1360.

VICSEK, T. 2001. Fluctuations and scaling in biology. Oxford University Press, New York, p.16-24.

VLCEK, J. \& CHEUNG, E. 1985. Fractal analysis of leaf shape. Canadian Journal of Forest Research 16:124-127.

WOLFRAM, S. 1984. Cellular automata as models of complexity. Nature 311:419-424. 